\title{
Long-Term Control of Endemic Hospital-Wide Methicillin-Resistant Staphylococcus aureus (MRSA): The Impact of Targeted Active Surveillance for MRSA in Patients and Healthcare Workers
}

\author{
Jesús Rodríguez-Baño, MD, PhD; Lola García, RN; Encarnación Ramírez, MD, PhD; Carmen Lupión, RN; \\ Miguel A. Muniain, MD, PhD; Carmen Velasco, PhD; Juan Gálvez, MD; M. Dolores del Toro, MD, PhD; \\ Antonio B. Millán, MD, PhD; Lorena López-Cerero, MD, PhD; Alvaro Pascual, MD, PhD
}

ов јестіve. To evaluate the long-term impact of successive interventions on rates of methicillin-resistant Staphylococcus aureus (MRSA) colonization or infection and MRSA bacteremia in an endemic hospital-wide situation.

DESIGN. Quasi-experimental, interrupted time-series analysis. The impact of the interventions was analyzed by use of segmented regression. Representative MRSA isolates were typed by use of pulsed-field gel electrophoresis.

Setting. A 950-bed teaching hospital in Seville, Spain.

patients. All patients admitted to the hospital during the period from 1995 through 2008.

METHODs. Three successive interventions were studied: (1) contact precautions, with no active surveillance for MRSA; (2) targeted active surveillance for MRSA in patients and healthcare workers in specific wards, prioritized according to clinical epidemiology data; and (3) targeted active surveillance for MRSA in patients admitted from other medical centers.

RESULTS. Neither the preintervention rate of MRSA colonization or infection ( 0.56 cases per 1,000 patient-days [ $95 \%$ confidence interval $\{C I\}, 0.49-0.62$ cases per 1,000 patient-days]) nor the slope for the rate of MRSA colonization or infection changed significantly after the first intervention. The rate decreased significantly to 0.28 cases per 1,000 patient-days $(95 \% \mathrm{CI}, 0.17-0.40$ cases per 1,000 patient-days) after the second intervention and to 0.07 cases per 1,000 patient-days ( $95 \% \mathrm{CI}, 0.06-0.08$ cases per 1,000 patient-days) after the third intervention, and the rate remained at a similar level for 8 years. The MRSA bacteremia rate decreased by $80 \%$, whereas the rate of bacteremia due to methicillin-susceptible $S$. aureus did not change. Eighty-three percent of the MRSA isolates identified were clonally related. All MRSA isolates obtained from healthcare workers were clonally related to those recovered from patients who were in their care.

CONCLUSION. Our data indicate that long-term control of endemic MRSA is feasible in tertiary care centers. The use of targeted active surveillance for MRSA in patients and healthcare workers in specific wards (identified by means of analysis of clinical epidemiology data) and the use of decolonization were key to the success of the program.

Infect Control Hosp Epidemiol 2010; 31(8):786-795

Methicillin-resistant Staphylococcus aureus (MRSA) is a leading cause of healthcare-associated infection. Infections caused by MRSA are associated with increased cost and mortality. ${ }^{1,2}$ Rates of nosocomial infection caused by MRSA are alarmingly high in many countries and have been increasing worldwide over recent years. ${ }^{3-5}$ Control of MRSA transmission within the hospital environment is presently a challenge, particularly in endemic situations. The medical literature on the epidemiology and control of healthcare-associated MRSA is plentiful, although most studies focus on particular aspects, such as outbreak control, specific infection control measures, or specific wards. At the same time, there are limited data available evaluating the impact of comprehensive infection control programs on hospital-wide MRSA or the long-term efficacy of infection control measures in endemic situations. ${ }^{6-10}$ Data from Spanish multicenter studies have shown that there was a significant increase in the prevalence of MRSA colonization or infection from $1994(16 \%)$ to $2002(31 \%)^{5}$ but that the prevalence reached a plateau at $29 \%$ in $2006 .{ }^{11}$ Data from the European Antimicrobial Resistance Surveillance System — which includes data from bacteremic episodes - have shown that MRSA prevalence has been stable since 2000 , at around $27 \% .^{12}$

From the Sección de Enfermedades Infecciosas (J.R.B., L.G., C.L., M.A.M., J.G., M.D.d.T., A.B.M.) and the Servicio de Microbiología (E.R., L.L.-C., A.P.), Hospital Universitario Virgen Macarena, and the Departamento de Medicina (J.R.-B., M.A.M., J.G.) and the Departamento de Microbiología (C.V., L.L.-C., A.P.), Universidad de Sevilla, Sevilla, Spain.

Received September 30, 2009; accepted February 9, 2010; electronically published June 4, 2010.

(C) 2010 by The Society for Healthcare Epidemiology of America. All rights reserved. 0899-823X/2010/3108-0003\$15.00. DOI: 10.1086/654003 
TA B LE 1. Summary of Our Study Evaluating the Long-Term Impact of Successive Interventions on Rates of Colonization or Infection due to Methicillin-Resistant Staphylococcus aureus (MRSA) and Rates of Bacteremia due to MRSA and Methicillin-Susceptible S. aureus (MSSA), at Hospital Universitario Virgen Macarena in Seville, Spain

\begin{tabular}{|c|c|}
\hline Variable & Definition \\
\hline Setting & 950-bed, acute care referral tertiary center serving a population of 550,000 \\
\hline Infection control team & $\begin{array}{l}\text { Hospital epidemiologist, infectious diseases physician, and microbiologist (all part time); } 3.5 \text { full- } \\
\text { time-equivalent infection control nurses. }\end{array}$ \\
\hline Entire study period & January 1995-December 2008 \\
\hline Characteristics of patient population & All admitted patients ( $\sim 40,000$ per year $)$ in endemic MRSA setting \\
\hline Design & Retrospective interrupted time series analyzed by use of segmented regression models \\
\hline \multicolumn{2}{|l|}{ Intervention } \\
\hline First & $\begin{array}{l}\text { Use of contact precautions for all patients colonized or infected with MRSA and use of cleaning } \\
\text { and disinfection protocol (all implemented in January 1997). }\end{array}$ \\
\hline Second & $\begin{array}{l}\text { Identification of wards with MRSA transmission, based on clinical epidemiology; active surveil- } \\
\text { lance for MRSA in colonized patients and healthcare workers in these wards; and decoloniza- } \\
\text { tion (all implemented in January 1999). }\end{array}$ \\
\hline Third & $\begin{array}{l}\text { Active surveillance for MRSA in colonized patients readmitted or admitted from long-term care } \\
\text { facilities or other hospitals (implemented January 2001). }\end{array}$ \\
\hline \multicolumn{2}{|l|}{ Study period } \\
\hline Period A & January 1995-December 1996 (preintervention period) \\
\hline Period B & January 1997-December 1998 \\
\hline Period C & January 1999-December 2000 \\
\hline Period D & January 2001-December 2008 \\
\hline Outcome measures & $\begin{array}{l}\text { Bimonthly incidences of healthcare-associated colonization or infection due to MRSA and } \\
\text { healthcare-associated bacteremia due to MRSA and MSSA }\end{array}$ \\
\hline
\end{tabular}

Several guidelines have been published with recommendations for controlling the spread of MRSA. ${ }^{13-16}$ MRSA control measures are complex, costly, and time-consuming. Because there remains some uncertainty about the best approach for preventing and controlling the spread of endemic hospitalwide MRSA, ${ }^{17}$ many hospitals probably do not devote enough resources to infection control activities, and there is wide variation in the extent of implementation of infection control programs. $^{18-21}$

In our hospital, no control measures for MRSA were undertaken until 1997. Since then, a comprehensive hospitalwide MRSA control program has been implemented in different phases. Our objective was to evaluate the impact of different bundles of MRSA control measures on the evolution of rates of MRSA colonization or infection and on the evolution of rates of bacteremia due to MRSA.

\section{METHODS}

We followed the ORION (Outbreak Reports and Intervention studies Of Nosocomial infection) statement for transparent reporting of intervention studies concerning nosocomial infections. $^{22}$ A summary of methods can be found in Table 1.

\section{Study Site}

The Hospital Universitario Virgen Macarena in Seville, Spain, is a 950-bed teaching hospital that receives approximately 40,000 admissions every year. In wards without an intensive care unit, $70 \%$ of the rooms have 3 beds, and $30 \%$ are private; the intensive care units had an open structure during the study period. An active global infection control program was implemented in 1995 that included the review and implementation of infection control protocols, the use of educational sessions, and the regular reporting of surveillance results. Also in 1995, a successful program using the bundle approach to control endemic Acinetobacter baumannii was implemented. ${ }^{23}$ The antibiotic policy did not change during the study period.

\section{Study Design}

To investigate the effects of the successive interventions specifically implemented to control the spread of MRSA, a retrospective, quasi-experimental, interrupted time-series study was used. The epidemiology of MRSA during the study period was characterized by use of basic clinical epidemiological data and molecular techniques.

\section{Study Periods and Interventions}

To evaluate the impact of 3 successive bundles of interventions, 4 periods were studied: period A (the preintervention period from January 1995 to December 1996), period B (the first intervention period from January 1997 to December 1998), period C (the second intervention period from January 1999 to December 2000), and period D (the third intervention period from January 2001 to December 2008). Likewise, hand 
тав в 2. Data on Case Mix, Antibiotic Use, and Epidemiology of Colonization or Infection due to Methicillin-Resistant Staphylococcus aureus (MRSA) and Bacteremia due to MRSA and Methicillin-Susceptible S. aureus (MSSA) in Patients at Hospital Universitario Virgen Macarena in Seville, Spain, during 4 Study Periods

\begin{tabular}{|c|c|c|c|c|}
\hline Variable & $\begin{array}{c}\text { Period A } \\
(1995-1996)\end{array}$ & $\begin{array}{c}\text { Period B } \\
(1997-1998)\end{array}$ & $\begin{array}{c}\text { Period C } \\
(1999-2000)\end{array}$ & $\begin{array}{c}\text { Period D } \\
(2001-2008)\end{array}$ \\
\hline Mean age of admitted patients ( $95 \% \mathrm{CI})$, years & $50(48-53)$ & $52(49-52)$ & $53(52-55)$ & $54(53-55)$ \\
\hline Mean no. of diagnoses per patient $(95 \% \mathrm{CI})$ & $1.49(1.44-1.55)$ & $1.52(1.47-1.57)$ & $1.75(1.62-1.83)$ & $1.74(1.70-1.77)$ \\
\hline \multicolumn{5}{|l|}{ Antibiotic consumption, DDDs per 100 patient-days } \\
\hline Total & 46 & 46 & 47 & 48 \\
\hline Fluoroquinolones & 1.6 & 2.6 & 4.2 & 5.6 \\
\hline Third-generation cephalosporins & 11.6 & 11.5 & 12.1 & 10.5 \\
\hline Glycopeptides & 7.8 & 7.9 & 4.8 & 4.9 \\
\hline $\begin{array}{l}\text { Mean no. of new cases of MRSA colonization or infec- } \\
\text { tion, per year }\end{array}$ & 190 & 195 & 76 & 65 \\
\hline $\begin{array}{l}\text { Mean proportion (\%) of new cases of MRSA colonization } \\
\text { or infection, per year, detected by use of clinical }\end{array}$ & & & & \\
\hline sample & $190 / 190(100)$ & 193/195 (99) & $43 / 76(57)^{\mathrm{a}}$ & $34 / 65(52)^{\mathrm{a}}$ \\
\hline $\begin{array}{l}\text { Mean proportion (\%) of new cases of MRSA coloniza- } \\
\text { tion, per year, detected by use of surveillance sample }\end{array}$ & 0 & 2/195 (1) & $32 / 76(42)^{\mathrm{a}}$ & $26 / 65(40)^{\mathrm{a}}$ \\
\hline $\begin{array}{l}\text { Mean proportion }(\%) \text { of new cases of healthcare-associ- } \\
\text { ated MRSA colonization or infection detected in the } \\
\text { first } 3 \text { calendar days of hospital admission }\end{array}$ & $8 / 190(4)$ & $10 / 195(5)$ & $7 / 76(9)$ & $8 / 65(12)^{b}$ \\
\hline $\begin{array}{l}\text { Mean proportion }(\%) \text { of new cases of MRSA coloniza- } \\
\text { tion, per year, admitted from other healthcare } \\
\text { centers }\end{array}$ & 0 & 0 & $1 / 76(1)$ & $5 / 65(8)^{c}$ \\
\hline $\begin{array}{l}\text { Mean bimonthly no. of new cases of healthcare-associated } \\
\text { MRSA colonization or infection, per } 1,000 \text { patient- } \\
\text { days }(95 \% \mathrm{CI})\end{array}$ & $0.56(0.49-0.62)$ & $0.55(0.48-0.61)$ & $0.28(0.17-0.40)$ & $0.07(0.06-0.08)$ \\
\hline $\begin{array}{l}\text { Mean bimonthly percentage of methicillin resistance } \\
\text { among healthcare-associated S. aureus isolates (95\% }\end{array}$ & & & & \\
\hline $\mathrm{CI})$ & $47(37-54)$ & $48(38-55)$ & $25(19-30)^{\mathrm{a}}$ & $11(8-14)^{\mathrm{a}, \mathrm{c}}$ \\
\hline $\begin{array}{l}\text { Mean bimonthly no. of new cases of healthcare-associated } \\
\text { bacteremia due to MRSA, per } 1,000 \text { patient-days } \\
(95 \% \mathrm{CI})\end{array}$ & $0.10(0.08-0.12)$ & $0.10(0.08-1.13)$ & $0.04(0.03-0.05)$ & $0.02(0.0-0.03)$ \\
\hline $\begin{array}{l}\text { Mean bimonthly no. of new cases of healthcare-associated } \\
\text { bacteremia due to MSSA, per 1,000 patient-days } \\
(95 \% \mathrm{CI})\end{array}$ & $0.12(0.10-0.13)$ & $0.11(0.10-0.13)$ & $0.11(0.10-0.13)$ & $0.12(0.11-0.13)$ \\
\hline
\end{tabular}

NOTE. $P$ values were determined by comparing percentages. CI, confidence interval; DDDs, defined daily doses.

${ }^{a} P<.01$ (by Fisher exact test), compared with periods A and B.

${ }^{\mathrm{b}} P=.03$, compared with period $\mathrm{A}$, and $P=.04$, compared with period $\mathrm{B}$.

c $P<.01$ (by Fisher exact test), compared with period C.

hygiene was promoted during the period from 1995 through 2008 by use of educational sessions and annual campaigns. Staffing levels did not change during the study period. During period A, no specific measures were undertaken for patients with MRSA colonization or infection. The 3 successive interventions were as follows:

First intervention (period B). Contact precautions were implemented in January 1997 for all patients colonized or infected with MRSA, according to the recommendations of the Centers for Disease Control and Prevention. ${ }^{24}$ Noncritical patient care equipment was dedicated to individual patients or cohorts. These patients were detected only via clinical sam- ples because active surveillance was not performed. Patients were placed in individual rooms (conventional wards) or in cohorts in open-structure intensive care units. Adherence to contact precautions was supervised and reinforced on a daily basis by an infection control nurse. Contact precautions were also implemented during all diagnostic or therapeutic maneuvers. A strict cleaning policy, including disinfecting all devices that came in contact with the patients, was also implemented; sodium hypochlorite was used to clean surfaces, and $70 \%$ ethyl alcohol was used to clean the noncritical medical equipment. ${ }^{25}$ All of these measures were maintained during the successive intervention periods. 
Second intervention (period $C$ ). In units where there was transmission of MRSA, active surveillance for MRSA in patients and healthcare workers (HCWs) and decolonization therapy were implemented in January 1999. Because the rates of colonization or infection did not change with the use of the previous MRSA control measures, active surveillance of MRSA colonization in patients and HCWs was performed in units with ongoing MRSA transmission. These units were identified as follows. The epidemiological data on all patients colonized or infected with MRSA during the preceding year were analyzed. The ward where the patient was first hospitalized and where MRSA colonization or infection was first detected was considered to be the location of acquisition; any other ward or healthcare facility where the patient had been admitted to or cared for in the preceding 2 years was also considered to be the possible location of acquisition. Wards and healthcare facilities with more than 1 attributable case of MRSA colonization during a 3-month period were considered as having ongoing MRSA transmission. Active surveillance was initiated in these wards successively, prioritizing those with the highest number of assigned cases. Active surveillance involved obtaining screening samples from all admitted patients to detect MRSA colonization, both at admission and every week thereafter, until no new cases were detected during a 2-week period. Also, all HCWs attending specific wards-including HCWs with frequent patient contact who come from outside the hospital, such as physiotherapists-were screened before they started their shift. Screening samples obtained from patients comprised nasal and perineal swab samples, open wound swab samples, respiratory tract samples from patients receiving mechanical ventilation or with chronic respiratory disease, and urine samples from catheterized patients. Only nasal swab samples were obtained from HCWs. This process was reinitiated whenever 2 or more cases were detected again separated by less than a month. Roommates of patients with MRSA colonization were also screened in wards where there was no active surveillance. All the measures adopted in the first intervention (ie, period B) were also performed.

Every patient with MRSA colonization was evaluated for application of the decolonization protocol; this involved applying mupirocin nasal ointment 3 times per day, plus daily body washing with $2 \%$ chlorhexidine gluconate for 5 days. The decolonization protocol was applied to patients without open, colonized wounds or respiratory tract colonization, without receipt of mechanical ventilation or a nasogastric tube, without urinary tract colonization in the presence of a urinary catheter, or without high-level mupirocin resistance. HCWs with MRSA colonization followed the same decolonization protocol. Nasal swab samples were obtained from colonized HCWs 1 week after decolonization therapy, to check for decolonization.

Third intervention (period D). Active surveillance for MRSA in readmitted patients previously colonized with MRSA and patients admitted from other healthcare centers was imple- mented in January 2001. In addition to the implementation of all the previously mentioned intervention measures, the third intervention required that all patients admitted from other hospitals or from long-term care facilities and all readmitted patients previously colonized with MRSA also be screened by use of the same protocol. Only readmitted patients previously colonized with MRSA were placed under preemptive isolation precautions. In addition, patients who had not been decolonized during hospitalization were reevaluated in an outpatient office, after hospital discharge, for decolonization therapy. Dispensers containing alcohol solution for hand hygiene were installed in all rooms in November 2000. Alcohol hand rubs had not been previously used in our healthcare center, but since November 2000, annual educational sessions have been used to promote hand hygiene with these products.

\section{Microbiological Testing}

S. aureus isolates were first identified by use of the MicroScan system (Dade Behring) or the Vitek 2 system (bioMérieux) and confirmed by use of the API Staph system (bioMérieux). Antimicrobial susceptibility testing was performed by use of both the Vitek 2 system and microdilution, according to Clinical Laboratory Standards Institute (formerly known as the National Committee for Clinical Laboratory Standards) recommendations. Mupirocin susceptibility was determined in strains isolated from nasal swab samples. Methicillin resistance was determined by use of an oxacillin-salt agar screening test, according to Clinical Laboratory Standards Institute guidelines. Surveillance samples were transported at room temperature and processed within 1-3 hours after collection. Swab samples were placed in tubes containing $1 \mathrm{~mL}$ of tryptone soya broth with $6.5 \%$ sodium chloride for 24 hours and subcultured on blood agar plates. Results were typically available in 36-48 hours. The clonal relatedness of selected isolates was determined by use of pulsed-field gel electrophoresis (PFGE), as described elsewhere. ${ }^{26}$ Whole chromosomal DNA was digested with SmaI. The clonal relatedness of PFGE patterns was determined according to the interpretative criteria used by Tenover et al. ${ }^{27}$

\section{Variables and Definitions}

The main outcome measures were the bimonthly incidence of healthcare-associated MRSA colonization or infection and the bimonthly incidence of healthcare-associated bacteremia due to MRSA, both defined as new cases per 1,000 patientdays. Cases were considered healthcare associated if the first sample yielding MRSA had been obtained more than 3 calendar days after hospital admission or if the first sample yielding MRSA had been obtained from an ambulatory patient who has an identified association with recent healthcare delivery. ${ }^{28}$ For the calculation of incidences of MRSA colonization or infection, only patients who had MRSA isolated from clinical samples were included, because active surveil- 


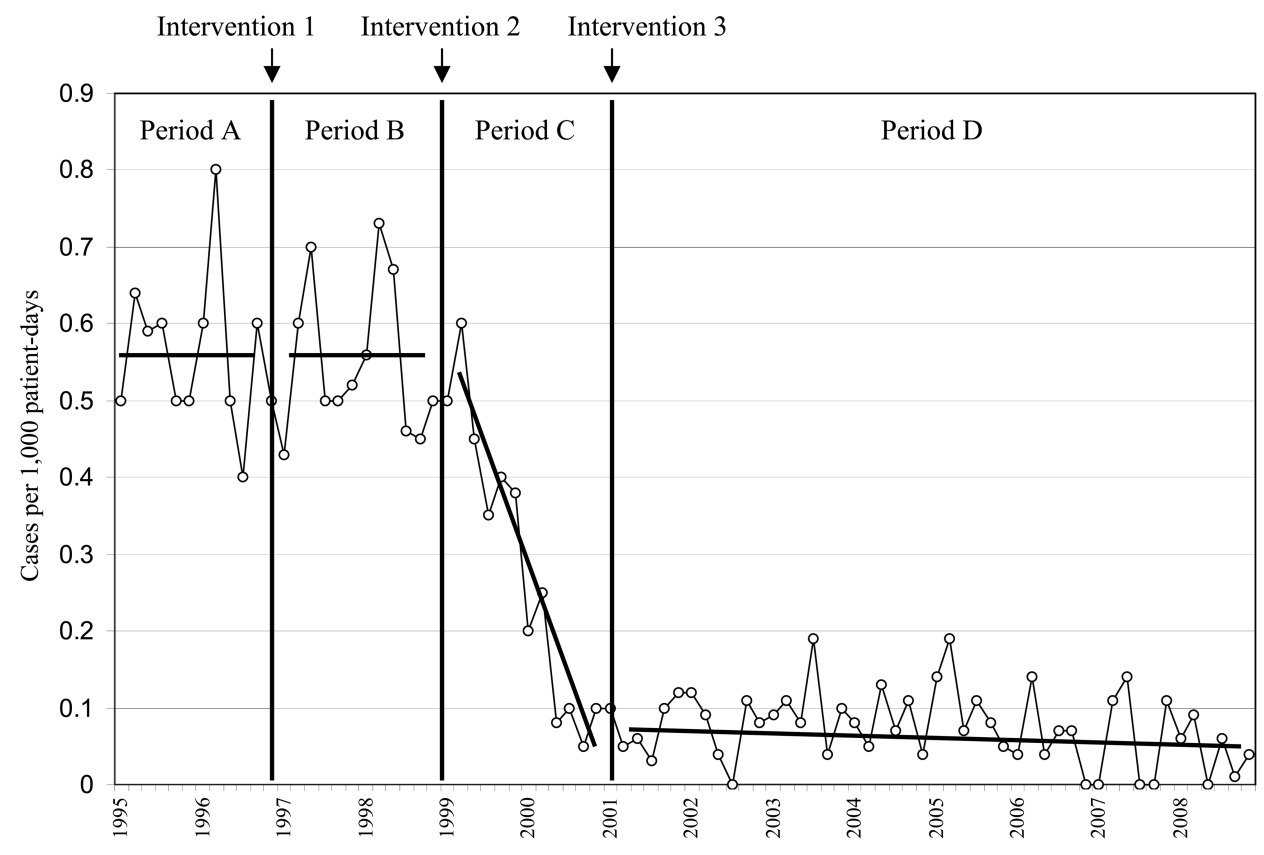

FIGURE 1. Bimonthly incidence rates of healthcare-associated methicillin-resistant Staphylococcus aureus colonization or infection during the 4 different study periods (from January 1995 to December 2008) at Hospital Universitario Virgen Macarena in Seville, Spain.

lance was not performed uniformly throughout the study periods.

\section{Statistical Analysis}

The effect of the successive interventions was analyzed by use of segmented regression analysis. ${ }^{29-31}$ In our study, we analyzed 3 interventions and 4 time periods (periods A-D); each time period was defined by the incidence rate at the beginning of the time period and the trend observed during the time period. Both the rapid and gradual effects of the interventions can be estimated by comparing the incidence rate and the trend of a period with those of the preceding period. The following model was fitted: $Y=\beta_{0}+\left(\beta_{1} \times\right.$ time $)+\left(\beta_{2}\right.$ $\times$ first intervention $)+\left(\beta_{3} \times\right.$ time after first intervention $)$ $+\left(\beta_{4} \times\right.$ second intervention $)+\left(\beta_{5} \times\right.$ time after second intervention $)+\left(\beta_{6} \times\right.$ third intervention $)+\left(\beta_{7} \times\right.$ time after third intervention) $+e$, where $Y$ represents the incidence of MRSA colonization or infection; $\beta_{0}$ represents the baseline incidence rate; $\beta_{1}$ represents the change in the incidence rate that occurred before implementation of the interventions; $\beta_{2}$, $\beta_{4}$, and $\beta_{6}$ represent the changes in the incidence rate immediately after implementation of the first, second, and third interventions, respectively; $\beta_{3}, \beta_{5}$, and $\beta_{7}$ represent the changes in the trend after implementation of each intervention, compared with the trend before implementation of the intervention; and $e$ represents the error term. The model was used to calculate the absolute change in incidence rate at specific time points. Serial autocorrelation was studied by visual inspection of residuals plotted against time and by use of the Durbin-Watson statistic, for which values close to 2.00 indicate no serious autocorrelation. ${ }^{29}$

We could not incorporate data on potential confounders to the model, because bimonthly data on these confounders were not available. We opted therefore to analyze several types of data sets, to provide an indirect control for confounding. Annual prevalence data concerning age and number of diagnoses were used to evaluate potential differences in case mix across the study periods and were obtained from yearly point-prevalence studies. ${ }^{32}$ Antimicrobial consumption (measured as defined daily doses per 1,000 patient-days per year) were obtained from the hospital pharmacy database. The incidence of healthcare-associated bacteremia due to methicillin-susceptible S. aureus (MSSA) was used to control for the potential confounding effect of changes in the number of blood culture samples obtained and in other infection control measures not specifically aimed at controlling MRSA. Categorical variables (eg, rate of methicillin resistance, number of patients with MRSA colonization detected by means of active surveillance, and number of wards with patients with MRSA colonization or infection) were compared by use of the $\chi^{2}$ test or the Fisher exact test, when necessary.

\section{RES ULTS}

\section{Patients}

During the 13-year study period (from 1995 to 2008), MRSA was isolated from clinical samples obtained from 1,230 patients. Of these patients, $824(67 \%)$ were considered to have 

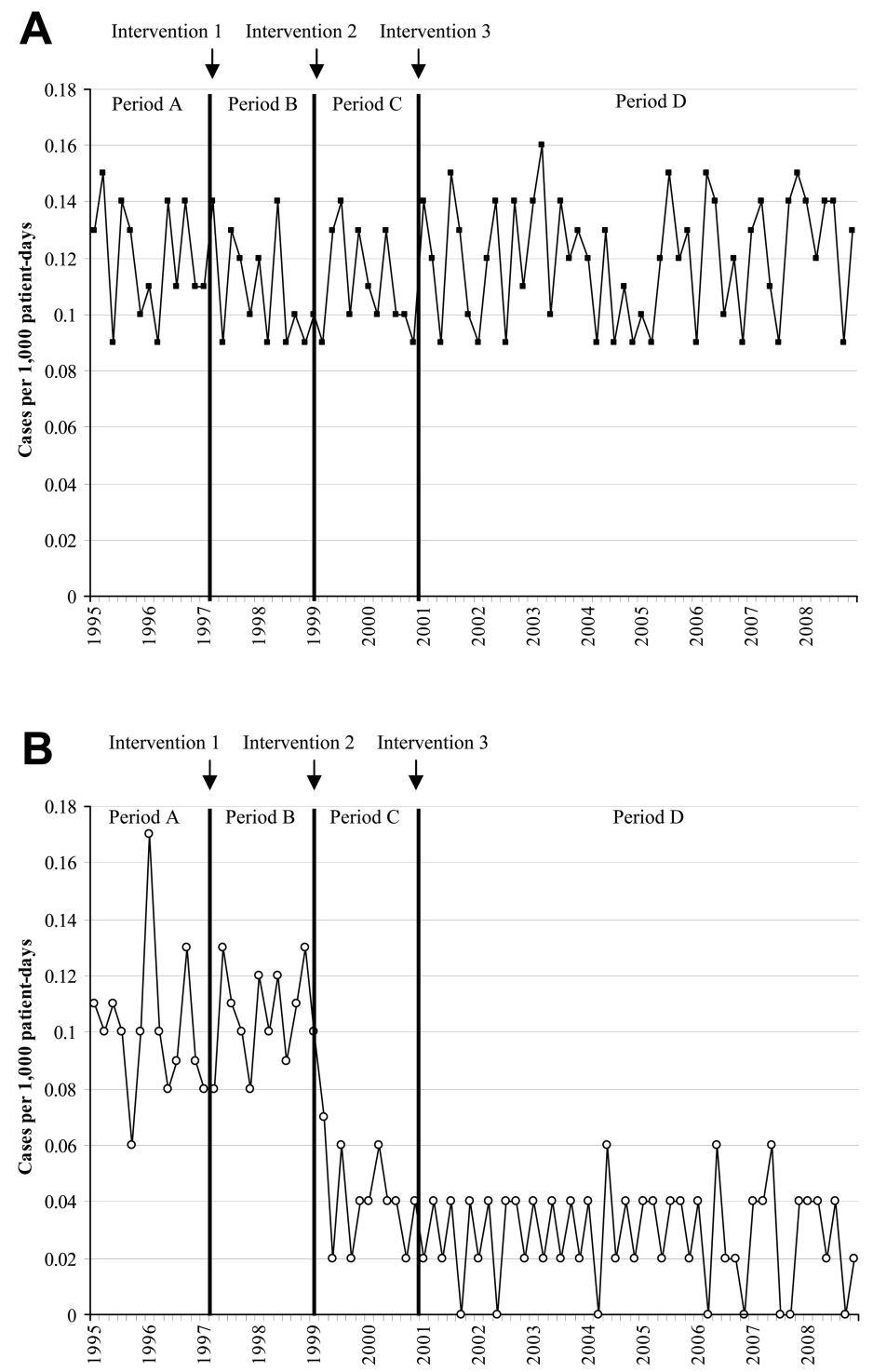

FIGURE 2. Bimonthly incidence rates of healthcare-associated bacteremia due to $(A)$ methicillin-susceptible and (B) methicillin-resistant Staphylococcus aureus during the 4 different study periods (from January 1995 to December 2008) at Hospital Universitario Virgen Macarena in Seville, Spain.

an infection due to MRSA, and of these patients, $214(26 \%)$ had MRSA bacteremia. Of the 1,230 patients, 359 (29\%) were admitted to adult medical services, 406 (33\%) were admitted to adult surgical services, $455(37 \%)$ were admitted to the adult intensive care unit, and $10(1 \%)$ were admitted to the pediatric ward. The in-hospital crude mortality rate was $40 \%$ (492 patients died). In addition, MRSA was isolated from another 306 patients by means of surveillance samples only.

Data regarding the case mix of admitted patients during the study periods are summarized in Table 2 . The mean age of admitted patients, the mean number of diagnoses per pa- tient, and antibiotic consumption all tended to increase over the course of the 13-year study period.

\section{Analysis of the Impact of Interventions}

Table 2 shows the mean numbers of cases (ie, incidence rates, along with 95\% confidence intervals) of colonization or infection due to MRSA and of bacteremia due to MSSA and MRSA during the 4 different study periods (periods A-D). The percentage of patients detected by means of active surveillance significantly increased during periods $C$ and $D$, and the percentage of patients admitted from another healthcare 
та вLE 3. Results of Segmented Regression Analysis of Incidences of Colonization or Infection due to Methicillin-Resistant Staphylococcus aureus (MRSA) and of Bacteremia due to MRSA and Methicillin-Susceptible S. aureus (MSSA), at Hospital Universitario Virgen Macarena in Seville, Spain, 1995-2008

\begin{tabular}{|c|c|c|c|c|c|c|}
\hline \multirow[b]{2}{*}{ Value } & \multicolumn{2}{|c|}{ MRSA colonization or infection } & \multicolumn{2}{|l|}{ MRSA bacteremia } & \multicolumn{2}{|l|}{ MSSA bacteremia } \\
\hline & Coefficient (95\% CI) & $P$ & Coefficient (95\% CI) & $P$ & Coefficient (95\% CI) & $P$ \\
\hline$\beta_{0}$ & $0.586(0.498-0.674)$ & $<.001$ & $0.100(0.076-0.124)$ & $<.001$ & $0.120(0.093-0.148)$ & $<.001$ \\
\hline$\beta_{1}$ & $-0.004(-0.16$ to 0.008$)$ & .4 & $0.001(-0.003$ to 0.004$)$ & .75 & $0.001(-0.003$ to 0.005$)$ & .6 \\
\hline$\beta_{2}$ & $0.030(-0.088$ to 0.147$)$ & .5 & $-0.018(-0.050$ to 0.014$)$ & .2 & $-0.015(-0.052$ to 0.022$)$ & .4 \\
\hline$\beta_{3}$ & $0.001(-0.016$ to 0.0180$)$ & .8 & $0.002(-0.003$ to 0.006$)$ & .4 & $-0.005(-0.01$ to 0.005$)$ & .9 \\
\hline$\beta_{4}$ & $0.065(-0.053$ to 0.182$)$ & .2 & $-0.051(-0.083$ to -0.020$)$ & .002 & $-0.009(-0.046$ to 0.028$)$ & 6 \\
\hline$\beta_{5}$ & $-0.045(-0.062$ to -0.029$)$ & $<.001$ & $-0.006(-0.010$ to -0.01$)$ & .01 & $-0.005(-0.005$ to 0.005$)$ & .9 \\
\hline$\beta_{6}$ & $0.077(-0.012$ to 0.165$)$ & .04 & $0.002(-0.022$ to 0.026$)$ & .8 & $0.001(-0.027$ to 0.029$)$ & .9 \\
\hline$\beta_{7}$ & $0.047(0.035-0.059)$ & $<.001$ & $0.003(0.000-0.006)$ & .05 & $-0.001(-0.005$ to 0.003$)$ & .7 \\
\hline
\end{tabular}

NOTE. $\beta_{0}$, baseline incidence; $\beta_{1}$, baseline trend; $\beta_{2}$, change in incidence after first intervention; $\beta_{3}$, change in trend after first intervention; $\beta_{4}$, change in incidence after second intervention; $\beta_{5}$, change in trend after second intervention; $\beta_{6}$, change in incidence after thir intervention; $\beta_{7}$, change in trend after third intervention; CI, confidence interval.

center who were detected by means of active surveillance significantly increased during period $\mathrm{D}$. The number of hospital wards with new cases of MRSA colonization or infection decreased from $28(90 \%)$ of 31 hospital wards in period A to $15(48 \%)$ of 31 hospital wards in period $\mathrm{D}(P<.001)$.

The evolution of the bimonthly incidences of colonization or infection due to MRSA, bacteremia due to MRSA, and bacteremia due to MSSA are shown in Figures 1 and 2. The results of the segmented regression analysis are shown in Table 3. With regard to cases of colonization or infection due to MRSA, the implementation of the first intervention (period B) caused no significant changes in the incidence rate or in the trend of incidence rates, compared with the preintervention period (period A). However, a very significant change in trend was observed after the implementation of the second intervention (period C); the change in the incidence rate was not significant because the effect of the second intervention only became noticeable after several months. The implementation of the third intervention (period D), when the incidence rate was less than 0.1 cases per 1,000 patient-days, caused no significant change in the incidence rate but was followed by a significant change in slope, which was still slightly downward during period D. With regard to cases of bacteremia due to MRSA, the implementation of the first intervention brought about no significant effect, whereas the implementation of the second intervention was followed by a significant change in the incidence rate and in the trend of incidence rates (Figure 2B). There were no significant changes in trend or rate for cases of bacteremia due to MSSA (Figure $2 A)$. There was no increase in the percentage of multidrugresistant gram-negative organisms (data not shown). We found no significant autocorrelation. As calculated for December 2000, the incidence of colonization or infection due to MRSA and the incidence of bacteremia due to MRSA had decreased from baseline by $83 \%$ and $80 \%$, respectively.

\section{Active Surveillance for MRSA in Patients and HCWs}

During periods $\mathrm{C}$ and $\mathrm{D}$, active surveillance was performed in 22 wards (more than once in 5 of them): 1 adult intensive care unit, 1 pediatric intensive care unit, 10 surgical wards, and 10 medical wards. More than $90 \%$ of patients and HCWs were screened in all wards. During active surveillance, 93\% of MRSA-colonized patients were detected by use of nasal and skin swab samples. In all wards where active surveillance was performed, 1 or more MRSA-colonized HCWs were detected (range, 1-5 HCWs). Overall, $36 \mathrm{HCWs} \mathrm{(30} \mathrm{nurses} \mathrm{and}$ 6 physicians) were colonized and treated according to the decolonization protocol, but they were not prevented from undertaking their clinical duties during treatment. Decolonization was effective in every HCW but one, as confirmed by follow-up screening; this HCW was a persistent carrier whose sample yielded a mupirocin-resistant isolate; therefore, this HCW agreed to be redeployed to a non-patient-care workplace. Implementation of active surveillance was followed by a rapid decrease in the number of new cases of MRSA colonization in every ward except urology, where there were 8 new cases the year after 2 MRSA-colonized HCWs had been identified; however, 7 of these patients had been admitted previously ( $1-3$ years before the HCWs were decolonized).

\section{Microbiological Testing}

Microbiological testing was performed on 90 selected MRSA isolates obtained from blood culture samples and representative of all study periods and all wards. Twelve antimicrobial resistance profiles were found; one profile (showing resistance to ciprofloxacin) accounted for $35 \%$ of isolates, and another profile (showing resistance to ciprofloxacin, erythromycin, and clindamycin) accounted for $37 \%$ of isolates.

By use of PFGE, 12 different clones were found, although 
$75(83 \%)$ of the 90 isolates were clonally related (clone A); this clone was predominant throughout the different study periods. Clonal relatedness was also studied in 5 different clusters of cases of MRSA colonization from wards with suspected ongoing MRSA transmission and included 20 clinical isolates, 30 surveillance isolates from patients, and 10 isolates from HCWs. In these 5 clusters, most of the isolates (range, $75 \%-100 \%$ ) from the patients were clonally related. The isolates that were recovered from HCWs belonged to 5 different clones and were indistinguishable in all cases from the predominant clones recovered from patients admitted to their wards.

\section{I S C U S S I O N}

Our results show that, in the context of the high rate of hospital-wide endemicity, implementing active surveillance for MRSA colonization in patients and HCWs, along with the contact precautions in specific wards, was very effective in decreasing rates of healthcare-associated colonization or infection due to MRSA and healthcare-associated bacteremia due to MRSA. Because MRSA bacteremia is associated with high mortality and morbidity, ${ }^{1}$ reducing the MRSA bacteremia rate is clinically relevant. The effect of the intervention cannot be explained by a clonal shift, changes in the case mix, or changes antimicrobial consumption; furthermore, the reduced rates were followed by a decrease in glycopeptide consumption; no other drug-resistant organism replaced MRSA. The reduction in the rates of MRSA colonization or infection in our healthcare center was the reverse of the overall situation in Spanish hospitals (particularly in Andalusia), with MRSA prevalence increasing significantly from 1993 to 2002 and stabilizing later at around 25\%. ${ }^{5,11,12}$ Communityacquired MRSA colonization or infection is still anecdotal in our area, ${ }^{5,11,33}$ so that significant bias due to misclassification of healthcare-associated MRSA colonization or infection is highly improbable. Whether such results are attainable only by applying measures to prevent nosocomial infections is open to debate; the fact that MRSA control was achieved in the context of an active global infection control program developed some years before argues against this.

We implemented several bundles of MRSA control measures at different time points and, therefore, had the opportunity to evaluate the additive impact of the different bundles. During the preintervention period, the incidences of MRSA colonization or infection and MRSA bacteremia were consistently high. Implementing contact precautions exclusively for patients who were identified by means of a clinical sample (during the first intervention) as being colonized or infected caused no significant change in the incidence rates. No incontrovertible evidence has been found that contact and isolation precautions alone are effective in controlling MRSA. ${ }^{34}$ Clinical cultures are known to identify only part of the patient population with MRSA colonization, ${ }^{35}$ and an unidentified reservoir (of mainly undetected MRSA-colonized pa- tients and HCWs) was probably key to endemic MRSA transmission.

Active surveillance for MRSA in MRSA-colonized patients and HCWs was introduced next, and this intervention was followed by a very significant reduction in incidence rates. Because mathematical modeling suggested that the incidence rates could be maintained by admitting MRSA-colonized patients in spite of adequate control, ${ }^{36}$ we later introduced surveillance for MRSA in patients admitted from other healthcare centers as well as readmitted patients. This measure did not reduce incidence rates further, and although it may have contributed to keeping them low, the workload and cost involved require closer analysis. Our approach during the third intervention (period D) was similar to the "search and destroy" strategy developed in the Netherlands, ${ }^{37}$ except for the fact that we did not implement preemptive contact precautions for patients admitted from other healthcare centers before the results of surveillance samples were made available.

After some years of debate, active surveillance is now recommended in most MRSA control guidelines. ${ }^{13-16}$ However, controversy exists regarding the efficacy of active surveillance in endemic situations, ${ }^{17,38}$ which microbiologic methods should be used, ${ }^{9}$ and whether active surveillance should be performed for all admitted patients or on targeted populations. ${ }^{8,9,38}$ Another interesting question is the importance of colonized HCWs in MRSA epidemiology in endemic situations, ${ }^{38}$ which may have been underestimated in some guidelines; our data do not provide definite evidence but do suggest that the role played by MRSA-colonized HCWs may have been significant in some wards. We point out that, because MRSA was endemic in all hospital wards, we prioritized active screening in wards with higher rates of MRSA transmission; these wards were identified by carefully analyzing data obtained by use of classic clinical epidemiology. Our results suggest that the hidden reservoir formed by colonized patients and HCWs played a key role in maintaining the endemicity of MRSA. These data were later confirmed by molecular analysis of selected isolates.

Our study has some limitations. The interventions were not implemented according to an advance program but successively after an interim analysis of the results, and our statistical analyses were performed retrospectively. We did not systematically assess compliance with contact precautions or the effectiveness of decolonization in patients. Segmented regression allowed us to investigate the effect of the various interventions, although only indirect controls for potential confounding variables, such as antibiotic consumption or changes in the case mix, could be assessed. Although we were unable to control the potential impact of unnoticed interventions, the evolution of MSSA bacteremia rates provided indirect evidence against a substantial effect. We did not analyze the cost of the interventions. Finally, molecular typing was only performed on selected isolates. In conclusion, the results of our study suggest that control of endemic MRSA is possible even in large hospitals by implementing comprehensive control programs that include active surveillance for 
MRSA in patients and HCWs in hospital units where MRSA transmission has been identified after analysis of clinical epidemiological data.

\section{ACKNOWLEDGMENTS}

We thank M. Beltrán, PhD (Servicio de Farmacia, Hospital Universitario Virgen Macarena, Seville, Spain), for providing the antibiotic consumption data; A. D. Harris, MD (Department of Epidemiology and Preventive Medicine, University of Maryland, Baltimore, MD), for useful advice regarding the analysis; and all members of the Infection Control and Antibiotic Policy Committee at the Hospital Universitario Virgen Macarena for their collaboration in the program.

Financial support. Ministerio de Sanidad y Consumo; Instituto de Salud Carlos III-Fondo Europeo de Desarrollo Regional; Spanish Network for the Research in Infectious Diseases (grant REIPI RD06/0008); Fondo de Investigación Sanitaria (grant PI051019).

Potential conflicts of interest. All authors report no conflicts of interest relevant to this article.

Address reprint requests to Jesús Rodríguez-Baño, $\mathrm{MD}, \mathrm{PhD}$, Sección de Enfermedades Infecciosas, Hospital Universitario Virgen Macarena, Avda Dr Fedriani 3, Sevilla 41009, Spain (jesusrb@us.es).

\section{REFERENCES}

1. Cosgrove SE, Sakoulas G, Perencevich EN, Schwaber MJ, Karchmer AW Carmeli Y. Comparison of mortality associated with methicillin-resistant and methicillin-susceptible Staphylococcus aureus bacteremia: a metaanalysis. Clin Infect Dis 2003;36:53-59.

2. Cosgrove SE, Carmeli Y. The impact of antimicrobial resistance on health and economic outcomes. Clin Infect Dis 2003;36:1433-1437.

3. Tiemersma EW, Bronzwaer S, Lyytikäinen O, et al. Methicillin-resistant Staphylococcus aureus in Europe, 1999-2002. Emerg Infect Dis 2004;10: 1627-1634.

4. Centers for Disease Control and Prevention. National Nosocomial Infections Surveillance (NNIS) system report, data summary from January 1992 through June 2004, issued October 2004. Am J Infect Control 2004;32:470-485.

5. Cuevas O, Cercenado E, Vindel A, et al. Evolution of the antimicrobial resistance of Staphylococcus spp. in Spain: five nationwide prevalence studies, 1986 to 2002. Antimicrob Agents Chemother 2004;48:4240-4245.

6. Tomic V, Sorli PS, Trinkaus D, Sorli J, Widmer A, Trampuz A. Comprehensive strategy to prevent nosocomial spread of methicillin-resistant Staphylococcus aureus in a highly endemic setting. Arch Intern Med 2004;164:2038-2043.

7. Huang SS, Yokoe DS, Hinrichsen VL, et al. Impact of routine intensive care unit surveillance cultures and resultant barrier precautions on hospital-wide methicillin-resistant Staphylococcus aureus bacteremia. Clin Infect Dis 2006;43:971-978.

8. Chaberny IF, Schwab F, Ziesing S, Suerbaum S, Gastmeier P. Impact of routine surgical ward and intensive care unit admission surveillance culture on hospital-wide nosocomial methicillin-resistant Staphylococcus aureus infections in a university hospital: an interrupted time-series analysis. J Antimicrob Chemother 2008;62:1422-1429.

9. Robicsek A, Beaumont JL, Paule SM, et al. Universal surveillance for methicillin-resistant Staphylococcus aureus in 3 affiliated hospitals. Ann Intern Med 2008;148:409-418.

10. Chowers MY, Paitan Y, Sheva Gottesman B, Gerber B, Ben-Nissan Y, Shitrit P. Hospital-wide methicillin-resistant Staphylococcus aureus control program: a 5 year follow-up. Infect Control Hosp Epidemiol 2009;30: 778-781.

11. Cuevas O, Cercenado E, Goyanes MJ, et al. Staphylococcus spp. en España: situación actual y evolución de la resistencia a antimicrobianos (19862006). Enferm Infecc Microbiol Clin 2008;26:269-277.

12. European Antimicrobial Resistance Surveillance System. EARSS Annual Report 2008. http://www.rivm.nl/earss/Images/EARSS\%202008_final _tcm61-65020.pdf. Accessed January 15, 2010.

13. Muto CA, Jernigan JA, Ostrowsky BE, et al. SHEA guideline for preventing nosocomial transmission of multidrug-resistant strains of Staphylococcus aureus and Enterococcus. Infect Control Hosp Epidemiol 2003;24: 362-386.

14. Coia JE, Duckworth GJ, Edwards DI, et al; Joint Working Party of the British Society of Antimicrobial Chemotherapy; Hospital Infection Society; Infection Control Nurses Association. Guidelines for the control and prevention of methicillin-resistant Staphylococcus aureus (MRSA) in healthcare facilities [published correction appears in J Hosp Infect 2006;64(1):97-98]. J Hosp Infect 2006;63(suppl 1):S1-S44.

15. Siegel JD, Rhinehart E, Jackson M, Chiarello L; Healthcare Infection Control Practices Advisory Committee. Management of multidrug-resistant organisms in health care settings, 2006. Am J Infect Control 2007; 35(10 suppl 2):S165-S193.

16. Rodríguez-Baño J, Bischofberger C, Alvarez-Lerma F, et al. Vigilancia y el control de Staphylococcus aureus resistente a meticilina en hospitales españoles. Documento de consenso GEIH-SEIMC y SEMPSPH. Enferm Infecc Microbiol Clin 2008;26:285-298.

17. Harbarth S. Control of endemic methicillin-resistant Staphylococcus aureus-recent advances and future challenges. Clin Microbiol Infect 2006; 12:1154-1162.

18. Preheim LC, Rimland D, Bittner MJ. Methicillin-resistant Staphylococcus aureus in Veterans Administration medical centers. Infect Control 1987; 8:191-194.

19. Hails J, Kwaku F, Wilson AP, Bellingan G, Singer M. Large variation in MRSA policies, procedures and prevalence in English intensive care units: a questionnaire analysis. Intensive Care Med 2003;29:481-483.

20. Richet HM, Benbachir M, Brown DE, et al. Are there regional variations in the diagnosis, surveillance, and control of methicillin-resistant Staphylococcus aureus? Infect Control Hosp Epidemiol 2003;24:334-341.

21. Rodríguez-Baño J, Millán AB, Domínguez MA, et al. Medidas de control de Staphylococcus aureus resistente a meticilina en hospitales españoles. Encuesta del proyecto SARM 2003 GEIH/GEMARA/REIPI. Enferm Infecc Microbiol Clin 2006;24:149-156.

22. Stone SP, Cooper BS, Kibbler CC, et al. The ORION statement: guidelines for transparent reporting of outbreak reports and intervention studies of nosocomial infection. Lancet Infect Dis 2007;7:282-288.

23. Rodríguez-Baño J, García L, Ramírez E, et al. Long-term control of hospital-wide endemic multidrug-resistant (MDR) Acinetobacter baumannii through a comprehensive "bundle" approach. Am J Infect Control 2009;37:715-722.

24. Garner JS. Guideline for isolation precautions in hospitals. The Hospital Infection Control Practices Advisory Committee [published correction appears in Infect Control Hosp Epidemiol 1996;17(4):214]. Infect Control Hosp Epidemiol 1996;17(1):53-80.

25. Sehulster LM, Chinn RYW, Arduino MJ, et al. Guidelines for environmental infection control in health-care facilities. Recommendations from CDC and the Healthcare Infection Control Practices Advisory Committee (HICPAC). Atlanta, GA: US Department of Health and $\mathrm{Hu}-$ man Services and Centers of Disease Control and Prevention, 2003. http://www.cdc.gov/ncidod/dhqp/pdf/guidelines/Enviro_guide_03.pdf. Accessed January 15, 2010.

26. Domínguez MA, Lencastre H, Liñares J, Tomasz A. Spread and maintenance of a dominant methicillin-resistant Staphylococcus aureus (MRSA) disease in a Spanish hospital. J Clin Microbiol 1994;32:2081-2087.

27. Tenover FC, Arbeit RD, Goering RV, et al. Interpreting chromosomal DNA restriction patterns produced by pulsed-field gel electrophoresis: criteria for bacterial strain typing. J Clin Microbiol 1995;33:2233-2239.

28. Cohen AL, Calfee D, Fridkin SK, et al. Recommendations for metrics for multidrug-resistant organisms in healthcare settings: SHEA/HICPAC position paper. Infect Control Hosp Epidemiol 2008;29:901-913. 
29. Wagner AK, Soumerai SB, Zhang F, Ross-Degnan D. Segmented regression analysis of interrupted time series studies in medication use research. J Clin Pharm Ther 2002;27:299-309.

30. Shardell M, Harris AD, El-Kamary SS, Furuno JP, Miller RM, Perencevich EN. Statistical analysis and application of quasi experiments to antimicrobial resistance intervention studies. Clin Infect Dis 2007;45:901907.

31. Raineri E, Crema L, De Silvestri A, et al. Meticillin-resistant Staphylococcus aureus control in an intensive care unit: a 10 year analysis. J Hosp Infect 2007;67:308-315.

32. Vaqué J, Roselló J, Arribas JL. Prevalence of nosocomial infections in Spain: EPINE study 1990-1997. EPINE Working Group. J Hosp Infect 1999;43(suppl):S105-S111.

33. Rodríguez-Baño J, Angeles Domínguez M, Blas Millán A, et al; GEIH/ GEMARA (SEIMC); REIPI. Clinical and molecular epidemiology of community-acquired, healthcare-associated and nosocomial methicillin-re- sistant Staphylococus aureus in Spain. Clin Microbiol Infect 2009;15(12): 1111-1118.

34. Cooper BS, Stone SP, Kibbler CC, et al. Isolation measures in the hospital management of methicillin resistant Staphylococcus aureus (MRSA): systematic review of the literature. BMJ 2004;329:533.

35. Salgado CD, Farr BM. What proportion of hospital patients colonized with methicillin-resistant Staphylococcus aureus are identified by clinical microbiological cultures? Infect Control Hosp Epidemiol 2006;27:116-121.

36. Bonten MJ, Austin DJ, Lipsitch M. Understanding the spread of antibiotic resistant pathogens in hospitals: mathematical models as tools for control. Clin Infect Dis 2001;33:1739-1746.

37. Vandenbroucke-Grauls CM. Methicillin-resistant Staphylococcus aureus control in hospitals: the Dutch experience. Infect Control Hosp Epidemiol 1996;17:512-513.

38. Lessing MPA, Jordens JZ, Bowler ICJ. When should healthcare workers be screened for methicillin-resistant Staphylococcus aureus? J Hosp Infect 1996;34:205-210. 